\title{
Reasons for Non-use of Proven Interventions for Hospital Inpatients: Pharmacists' Perspectives
}

\author{
Arden Barry, Peter Loewen, Jane de Lemos, and Karen G Lee
}

\begin{abstract}
Background: Recently, health care institutions have been using performance indicators to measure and improve quality of care. One such indicator, the Ideal Medication Intervention Index, reflects the rate of implementation of proven pharmacologic interventions, which studies have shown are underutilized. Identifying the reasons why proven interventions are underused is essential to determining how their rate of use can be improved.
\end{abstract}

Objective: To characterize the reasons for non-use of proven interventions from the perspective of clinical pharmacists within the authors' health care organization.

Methods: A survey of all clinical pharmacists within the organization was conducted. The survey used standardized, case-based scenarios involving pharmacologic interventions known to improve health outcomes. Respondents were asked to rank potential reasons why a patient might not receive a proven intervention.

Results: Of the 115 pharmacists invited, 53 (46\%) participated in the survey. Most of the respondents practised on medical wards. The 2 most common reasons for non-use of proven interventions were a team preference to defer management of such issues to the outpatient care provider and issues related to workload.

Conclusions: Clinical pharmacists revealed that their perceptions of priorities, communication with their interdisciplinary teams, and workload issues contributed to non-use of proven pharmacologic interventions among patients in their care. Efforts to increase the utilization of the proven clinical interventions studied here should focus on changing pharmacists' perceptions of priorities.

Key words: quality assessment in health care, patient safety, knowledge translation, therapeutic omission, guideline adherence, care gap

Can J Hosp Pharm 2009;62(5):381-385

\section{RÉSUMÉ}

Contexte : Récemment, les établissements de santé ont utilisé des indicateurs de rendement pour évaluer et améliorer la qualité des soins. L'un de ces indicateurs, l'indice d'intervention pharmacologique idéale, reflète le taux de mise en œuvre d'interventions pharmacologiques éprouvées, dont la sous-utilisation a été montrée par des études. La détermination des raisons pour lesquelles les interventions éprouvées sont sous-utilisées est essentielle pour définir comment on peut accroître leur utilisation.

Objectif : Caractériser les raisons de la non-utilisation des interventions éprouvées, du point de vue des pharmaciens cliniciens de l'établissement de santé des auteurs.

Méthodes : Un sondage de tous les pharmaciens cliniciens de l'établissement de santé a été effectué. Le sondage comportait des études de cas standardisées impliquant des interventions pharmacologiques connues pour améliorer les résultats cliniques. On a demandé aux répondants de classer les raisons potentielles de l'absence d'intervention éprouvée pour un patient.

Résultats : Des 115 pharmaciens invités à participer, 53 (46\%) ont répondu au sondage. La plupart des répondants travaillaient dans des unités de médecine. Les deux raisons les plus courantes pour l'absence d'interventions éprouvées étaient la préférence de l'équipe de relayer la prise en charge de tels problèmes de santé au fournisseur de soins de santé externe, et les questions liées à la charge de travail.

Conclusions : Les pharmaciens cliniciens ont révélé que leurs perceptions des priorités, la communication avec leurs équipes interdisciplinaires et les motifs liés à la charge de travail ont contribué à l'absence d'interventions pharmacologiques éprouvées dans les soins de leurs patients. Les efforts pour accroître le recours aux interventions cliniques éprouvées évaluées dans cette étude doivent s'attarder à changer les perceptions qu'ont les pharmaciens des priorités.

Mots clés : évaluation de la qualité des soins de santé, sécurité des patients, application des connaissances, omission thérapeutique, observance des lignes directrices, lacune dans les soins

[Traduction par l'éditeur] 


\section{INTRODUCTION}

Consensus guidelines have been developed in both the United States and Canada recommending the use of proven clinical interventions for various disease states, to improve patient safety and quality of care. Despite these publications, a large gap still exists between the number of patients who meet the criteria for use of these interventions and the number of patients who actually receive them. ${ }^{1,2}$ In previous work, we defined a proven intervention as a medical intervention that is generally accepted as being effective, for which the benefits outweigh the risks, and for which there is high-quality evidence in the published literature and/or reputable practice guidelines recommending its use. ${ }^{3}$ A recent patient safety initiative demonstrated that the majority of medical errors in a retrospective cohort analysis were errors of omission (e.g., patients receiving too little medical care), as opposed to errors of commission (e.g., overprescribing, adverse drug reactions). ${ }^{4}$

In recent years, health care institutions have been using performance indicators as tools to improve the quality of care provided. ${ }^{1,5-10}$ In addition, hospitals are now becoming accountable for their performance in delivering evidence-based treatments. ${ }^{5}$ In the United States, organizations such as the Joint Commission and the Hospital Quality Alliance require that hospitals report their performance in providing certain pharmacologic therapies as a component of accreditation. ${ }^{6,10}$ Identifying the reasons why proven interventions are underutilized, in the absence of contraindications, is essential to determining how the rate of implementation can be improved. Vancouver Coastal Health - Providence Health Care considered the rate of implementation of evidence-based pharmacologic interventions to be indicative of the quality of care that inpatients were receiving. Therefore, a formal quality-of-care indicator, the Ideal Medication Intervention (IMI) Index, was developed and implemented in 2006. This index was based on the rate of implementation of 9 proven pharmacologic interventions for 4 chronic disease states for general medicine inpatients at several acute care institutions in the region. The IMI Index reflects the proportion of cases in which a proven therapy has been implemented, relative to the number of cases with an indication for (and no contraindication to) that therapy. Examples of the interventions and conditions included in the IMI Index are use of $ß$-blockers, angiotensin-converting enzyme (ACE) inhibitors or angiotensin receptor blockers, and spironolactone in systolic heart failure; use of acetylsalicylic acid, ACE inhibitors, B-blockers, and statins in coronary artery disease; use of warfarin in atrial fibrillation; and use of bisphosphonates, calcium, and vitamin D in osteoporosis. Initial measurement of the IMI Index among general medical inpatients in 2006-2007 showed that the overall rate of implementation was $79 \%$, lower than the predefined goal of $90 \%$ and the potential achievement of $100 \%$.
We believe that clinical pharmacists can influence the rate of implementation of proven interventions by identifying intervention opportunities for the patients under their care. The purpose of this study was to identify opportunities for increasing utilization of proven pharmacologic interventions in hospital inpatients by characterizing, from the perspective of clinical pharmacists within our health care organization, why such interventions are underutilized for patients who have valid indications for them. To our knowledge, no published work addressing this question is available.

\section{METHODS}

The study was a survey-based assessment. The target population was all of the pharmacists with direct patient care responsibilities at any of the 4 main acute care institutions in Vancouver Coastal Health - Providence Health Care. This population also included a small cohort of pharmacists practising in residential care settings. An e-mail message was sent to each pharmacist, with an invitation to participate in an online survey. The survey presented 6 case-based scenarios, each of which explored a specific pharmacologic intervention (or group of interventions) that have shown to improve health outcomes in the context represented by the scenario. Respondents were instructed to select in rank order, from a list of 9 reasons, the 3 most common reasons why patients under their care might not receive the pharmacologic intervention or interventions discussed. There being no prior published work in this area, the 9 reasons presented in the questionnaire were developed by the authors on the basis of their own extensive clinical practice experience and were augmented and informally validated through consultation with a group of staff pharmacists in our institution. Respondents were invited to give open-ended responses at the end of the survey if they wished to augment or clarify any of their responses. The scenarios and questions were tested on a small cohort of the target sample to assess clarity, a process that resulted in minor modifications to the survey tool.

The responses for each case scenario were aggregated and weighted according to their rank order: 3 points for a first-place rank, 2 points for a second-place rank, and 1 point for a third-place rank. Differences in the distribution of responses by demographic characteristics were explored using appropriate inferential statistics.

The 6 case-based scenarios involved acute coronary syndrome, systolic heart failure, coronary artery disease, atrial fibrillation, smoking cessation, and osteoporosis. Five of the scenarios were based on the conditions included in the institution's pharmacotherapeutic quality improvement program efforts (i.e., the IMI Index), and the sixth (smoking cessation) was added because of a concurrent institution-wide effort to improve identification and intervention for patients who smoked. Because prior data indicated that intervention rates are 
higher for the condition most responsible for the patients' admission, all of the cases were designed so that the proven pharmacologic intervention did not relate to the patient's reason for admission. ${ }^{3}$ Also, respondents were instructed to assume that the patient had no contraindications to the therapy recommended. See Box 1 for a sample scenario.

\section{RESULTS}

A total of 115 invitations were sent, and 53 pharmacists participated in the survey ( $46 \%$ response rate). The demographic characteristics of respondents appear in Table 1. The response rates were roughly proportional to the numbers of pharmacists at each site. A summary of the weighted responses to the survey can be found in Table 2 .

Among pharmacists with less than 5 years' experience in their specified areas, the weighted frequency was significantly higher for "I do not have the opportunity to recognize problems like this due to workload and/or other priorities" and "I might identify this problem, but I do not have time to investigate the patient's history in enough detail to determine if contraindications exist" than among those with at least 5 years of experience. Among pharmacists with at least 5 years of experience, the weighted frequency was significantly higher for "I would likely recognize the problem but take no action as patients on my ward are typically non-adherent once discharged" than among those with less than 5 years experience.

Among respondents with a daily patient load of up to 30 , the weighted frequency was significantly higher for "I do not have the opportunity to recognize problems like this due to workload and/or other priorities", "I do not have a forum in which to discuss the patient's plan with the team (e.g., rounds) in order to identify and/or resolve possible problems", and "Even if I did identify a problem, and I know what to do, my team does not usually initiate these types of therapies despite my recommendations" than among those with daily patient loads above 30. Among respondents with a daily patient load above 50 the weighted frequency was significantly higher for "I would likely recognize the problem but take no action as patients on my ward are typically non-adherent once discharged" than among those with a lower daily patient load.

\section{DISCUSSION}

All clinical pharmacists within the organization were invited to participate in this survey. Therefore, the results reflect a wide variety of practices. We believe this allowed us to capture a representative cross-section of opinions based on experience, education, and training.

Of the reasons listed in the survey, the one with the greatest weighted frequency involved patients not receiving therapies because the interdisciplinary team preferred to leave such issues to the outpatient care provider. This finding implies that the pharmacist would not make any recommendations to the team because of this known preference or that the team would reject any recommendations that were made. A similar reason, whereby the pharmacist would communicate directly with the patient or the outpatient caregiver rather than addressing the issue with the team, had a lower weighted frequency. From this we inferred that initiating the therapies covered by this

\section{Box 1. Sample Case Scenario from Survey}

A 71-year-old female is admitted to your ward for a reason unrelated to cardiovascular disease. On day 5 of her hospital stay she experiences an acute episode of diaphoresis, shortness of breath, and tachycardia. A cardiac work-up is performed, and it is determined that the patient experienced a mild myocardial infarction. With respect to your practice, why might this patient NOT be started on the bundle of ACE inhibitor, beta-blocker, statin, and ASA for secondary CV prevention? Please rank up to three responses, if applicable.

1. I do not have the opportunity to recognize problems like this due to workload and/or other priorities.

2. I do not have a forum in which to discuss the patient's plan with the team (e.g., rounds) in order to identify and/or resolve possible problems.

3. I might identify this problem, but I do not have time to investigate the patient's history in enough detail to determine if contraindications exist.

4. I might identify this problem, but I am not confident I am familiar enough with the current treatment guidelines.

5. Even if I did identify a problem, and I know what to do, my team would normally prefer to leave this issue to the patient's outpatient caregiver to solve (general practitioner, specialist).

6. Even if I did identify a problem, and I know what to do, my team does not usually initiate these types of therapies despite my recommendations.

7. I would likely recognize the problem but take no action as I would assume/prefer that the patient's outpatient caregiver (general practitioner, specialist) takes care of the problem.

8. I would likely recognize the problem but would speak to the patient directly and/or communicate with the patient's outpatient caregiver (general practitioner, specialist) as opposed to addressing the problem with the team.

9. I would likely recognize the problem but take no action as patients on my ward are typically non-adherent once discharged (e.g., history of non-adherence, unable to afford medications). 
Table 1. Characteristics of 53 Clinical Pharmacists Responding to a Survey about Non-use of Proven Interventions

\begin{tabular}{lc} 
Characteristic & No. (\%) \\
\hline Primary practice area* & $43(81)$ \\
Medicine & 13 \\
General medicine & 8 \\
Long-term care or residential care & 7 \\
Emergency medicine & 5 \\
Family practice & 4 \\
Leukemia or stem cell transplantation & 4 \\
Intensive care unit & 4 \\
Nephrology & 4 \\
Psychiatry & 3 \\
Palliative care & 3 \\
Cardiology & 2 \\
Total parenteral nutrition & 2 \\
Solid organ transplantation & 1 \\
Respirology & 1 \\
Infectious diseases & 1 \\
Neurology & 1 \\
HIV & 1 \\
Pediatrics & $10(19)$ \\
Surgery & 6 \\
General surgery & 4 \\
Orthopedics & 2 \\
Vascular surgery & 2 \\
Other surgery &
\end{tabular}

Time in current primary practice area $(n=51)$

$<5$ years 30

$5-10$ years

10-20 years

$3(25)$

3 (6)

20-30 years

$5(10)$

\section{Daily patient load $(n=51)$}

$\begin{array}{lrr}<20 & 10 & (20) \\ 21-30 & 7 & (14) \\ 31-50 & 19 & (37) \\ 51-70 & 5 & (10) \\ >70 & 10 & (20)\end{array}$

*Participants could select more than one category.

survey during the inpatient stay was not a high priority for pharmacists. The basis for this lack of priority may need to be explored further or confronted, considering our perception that the therapies a patient is receiving when he or she is discharged from hospital may influence long-term management of the condition, and the evidence that inappropriately omitted therapies at the time of hospital discharge can contribute to adverse events after the hospital stay and readmission. ${ }^{11,12}$

Several of the reasons listed in the survey (i.e., reasons 5 through 9 in Box 1) could be explained by the pharmacist having become conditioned to adopt the preferences of the team. It is natural that a pharmacist who anticipates that the team is unlikely to accept a recommendation to initiate a proven therapy would not make the recommendation in the first place. This finding may be an opportunity to remind pharmacists of their obligation to identify drug-related problems on behalf of their patients. As a consequence, they must assume the role of patient advocate. Evidence suggests that it may be incorrect to assume that the primary care physician will close a therapeutic gap in chronic disease management. ${ }^{12}$

The reasons with the second- and third-highest weighted frequency were workload issues, whereby the respondents felt they did not have enough time to review the patient's history in sufficient detail to feel confident about their recommendations. From this we inferred that the respondents were indicating that the chronic disease therapy issues covered by the survey scenarios were subordinate in priority to other acute care issues or that they simply did not have enough time to deal with as many acute and chronic care issues as they would like. If the former were true, realignment of pharmacists' attitudes and priorities would be required to improve the rate of utilization. Only if the latter were true would devoting more pharmacist resources to the target patients be a remedy. These possibilities might be differentiated with another survey dealing only with acute care issues. If such a survey had a similar response rate for "I do not have the opportunity to recognize problems like this due to workload and/or other priorities," the inference that the pharmacists had insufficient time would be confirmed.

It appears that the respondents were relatively unconcerned about their training and knowledge base, about their patients not adhering with therapy after discharge, and about not having a forum (e.g., rounds) to discuss patient issues. Qualitative synthesis of the open-ended responses yielded only 2 themes: lack of time to make recommendations because of high patient turnover and competing demands, with priority given to other, more urgent issues.

These results lead us to believe that initiatives to improve utilization of proven interventions should focus on understanding pharmacists' perceptions of priorities and then improving the alignment of these priorities with patients' needs. Furthermore, the spectrum of drug-related problems encountered in the hospital should be viewed in terms of their effects on patients, their prevalence, the effort required to resolve them, and the benefits anticipated from doing so.

Some limitations of the study methodology are notable. To maximize the response rate, the case scenarios were highly structured, relatively brief, and limited in number. Even so, the $46 \%$ response rate was suboptimal, introducing the possibility of unknown sampling biases. We attempted to list enough possible reasons for non-use of proven interventions to allow respondents to select those that best reflected their practice, but some respondents indicated that they skipped certain cases because a suitable reason was not listed. This study also lacked power to explore potential interactions between demographic 
Table 2. Weighted Frequency of Reasons for Non-use of Proven Interventions

Reason for Non-use of Proven Intervention

Weighted Frequency (\%)

Even if I did identify a problem, and I know what to do, my team would normally prefer to leave

this issue to the patient's outpatient caregiver to solve (general practitioner, specialist).

I do not have the opportunity to recognize problems like this due to workload and/or other priorities.

31.6

I might identify this problem, but I do not have time to investigate the patient's history in enough detail

to determine if contraindications exist.

I would likely recognize the problem but would speak to the patient directly and/or communicate

with the patient's outpatient caregiver (general practitioner, specialist) as opposed to addressing

the problem with the team.

22.3

Even if I did identify a problem, and I know what to do, my team does not usually initiate these types

of therapies despite my recommendations.

I do not have a forum in which to discuss the patient's plan with the team (e.g., rounds) in order

to identify and/or resolve possible problems.

I would likely recognize the problem but take no action as patients on my ward are typically

non-adherent once discharged (e.g., history of non-adherence, unable to afford medications)

11.0

I would likely recognize the problem but take no action as I would assume/prefer that the patient's

outpatient caregiver (general practitioner, specialist) takes care of the problem.

I might identify this problem, but I am not confident I am familiar enough with the current

treatment guidelines.

3.1

1.6

0.5

characteristics and response distributions with multivariate regression methods.

This study demonstrated that the reasons, factors, and barriers related to lack of implementation of proven clinical therapies are complex and heterogeneous. Clinical pharmacists revealed that their perceptions of priorities, communication with their interdisciplinary teams, and workload issues contribute to non-use of proven pharmacologic interventions among patients in their care.

\section{References}

1. Fonarow GC, Yancy CW, Albert NM, Curtis AB, Stough WG, Gheorghiade $\mathrm{M}$, et al. Improving the use of evidence-based heart failure therapies in the outpatient setting: The IMPROVE HF performance improvement registry. Am Heart J 2007;154(1):12-38.

2. Tapson VF, Hyers TM, Waldo AL, Ballard DJ, Becker RC, Caprini JA, et al.; NABOR (National Anticoagulation Benchmark and Outcomes Report) Steering Committee. Antithrombotic therapy practices in US hospitals in an era of practice guidelines. Arch Intern Med 2005;165(13):1458-1464.

3. Huang C, Loewen P, Pelletier T, Slater J, Chung M. Implementation of proven interventions in general medical inpatients: development and evaluation of a new quality indicator for drug therapy. Qual Saf Health Care 2008;17(4):269-274.

4. Hayward RA, Asch SM, Hogan MM, Hofer TP, Kerr EA. Sins of omission: getting too little medical care may be the greatest threat to patient safety. J Gen Intern Med 2005;20(8):686-691.

5. Werner RM, Bradlow ET. Relationship between Medicare's hospital compare performance measures and mortality rates. JAMA 2006;296(22):2694-2702.

6. Isetts BJ, Brown LM, Schondelmeyer SW, Lenarz LA. Quality assessment of a collaborative approach for decreasing drug-related morbidity and achieving therapeutic goals. Arch Intern Med 2003;163(15):1813-1820.

7. Subramanian U, Sutherland J, McCoy KD, Welke KF, Vaughn TE, Doebbeling BN. Facility-level factors influencing chronic heart failure care process performance in a national integrated health delivery system. Med Care 2007;45(1):28-45.

8. Glickman SW, Ou FS, DeLong ER, Roe MT, Lytle BL, Mulgund J, et al. Pay for performance, quality of care, and outcomes in acute myocardial infarction. JAMA 2007;297(21):2373-2380.
9. Bradley EH, Herrim J, Mattera JA, Holmboe ES, Wang Y, Frederick P, et al. Quality improvement efforts and hospital performance: rates of betablocker prescription after acute myocardial infarction. Med Care 2005;43(3):282-292.

10. Jha AK, Li Z, Orav EJ, Epstein AM. Care in U.S. hospitals - the Hospital Quality Alliance Program. N Engl J Med 2005;353(3):265-274.

11. Forster AJ, Murff HJ, Peterson JF, Gandhi TK, Bates DW. The incidence and severity of adverse events affecting patients after discharge from the hospital. Ann Intern Med 2003;138(3):161-167.

12. Kripalani S, LeFevre F, Phillips CO, Williams MV, Basaviah P, Baker DW. Deficits in communication and information transfer between hospitalbased and primary care physicians: implications for patient safety and continuity of care. JAMA 2007;297(8):831-841.

Arden Barry, BSC, BSC(Pharm), ACPR, was, at the time of writing, with Vancouver Coastal Health - Providence Health Care Regional Pharmacy Services, Vancouver, British Columbia. He is now a Doctor of Pharmacy student at the University of British Columbia, Vancouver, British Columbia.

Peter Loewen, BSc(Pharm), ACPR, PharmD, is with Vancouver Coastal Health - Providence Health Care Regional Pharmacy Services, and the Faculty of Pharmaceutical Sciences, University of British Columbia, Vancouver, British Columbia.

Jane de Lemos, BPharm, PharmD, MSc, is with Vancouver Coastal Health - Providence Health Care Regional Pharmacy Services, and the Faculty of Pharmaceutical Sciences, University of British Columbia, Vancouver, British Columbia.

Karen G Lee, BSc(Pharm), ACPR, was, at the time of writing, with Vancouver Coastal Health - Providence Health Care Regional Pharmacy Services, Vancouver, British Columbia. She is now a medical student at the University of British Columbia, Vancouver, British Columbia.

\section{Address correspondence to:}

Dr Peter Loewen

Vancouver Coastal Health -

Providence Health Care Regional Pharmacy Services

3rd floor, 865 West 10th Avenue

Vancouver BC V5Z 1M9

e-mail: Peter.Loewen@vch.ca 